\title{
Unraveling the Radiative Pathways of Hot Carriers upon Intense Photoexcitation of Lead Halide Perovskite Nanocrystals
}

Paris Papagiorgis, ${ }^{\dagger}$ Andreas Manoli, ${ }^{\dagger}$ Sozos Michael, ${ }^{\dagger}$ Caterina Bernasconi, ${ }^{\ddagger}$, Maryna I. Bodnarchuk, $^{\S \odot}$ Maksym V. Kovalenko, ${ }^{*} \S_{(0)}$ Andreas Othonos, $"$ and Grigorios Itskos* ${ }^{* \dagger}$

${ }^{\dagger}$ Department of Physics, Experimental Condensed Matter Physics Laboratory, University of Cyprus, Nicosia 1678, Cyprus

${ }^{\ddagger}$ Institute of Inorganic Chemistry, Department of Chemistry and Applied Biosciences, ETH Zürich, CH-8093 Zürich, Switzerland

${ }^{\S}$ Laboratory for Thin Films and Photovoltaics, Empa - Swiss Federal Laboratories for Materials Science and Technology, Überlandstrasse 129, CH-8600 Dübendorf, Switzerland

"Department of Physics, Laboratory of Ultrafast Science, University of Cyprus, Nicosia 1678, Cyprus

Supporting Information

ABSTRACT: The slowdown of carrier cooling in lead halide perovskites (LHP) may allow the realization of efficient hot carrier solar cells. Much of the current effort focuses on the understanding of the mechanisms that retard the carrier relaxation, while proof-of-principle demonstrations of hot carrier harvesting have started to emerge. Less attention has been placed on the impact that the energy and momentum relaxation slowdown imparts on the spontaneous and stimulated light-emission process. LHP nanocrystals (NCs) provide an ideal testing ground for such studies as they exhibit bright emission and high
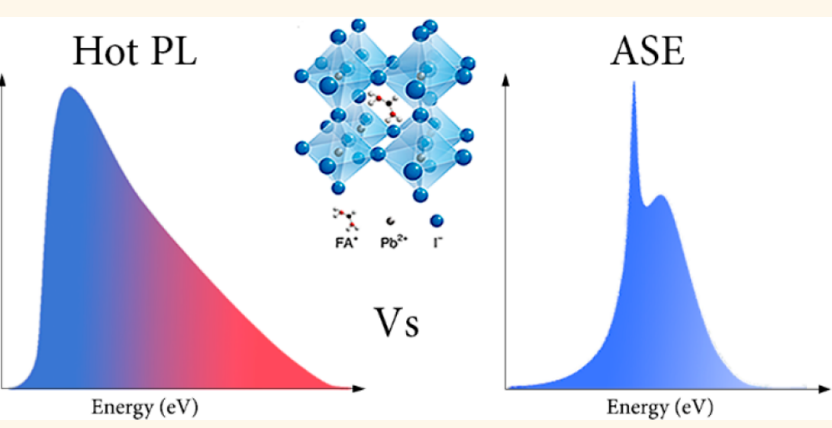
optical gain, while the carrier cooling bottleneck is further pronounced compared to their bulk analogues due to confinement. Herein, the luminescent properties of $\mathrm{CsPbBr}_{3}$, $\mathrm{FAPbBr}_{3}$, and $\mathrm{FAPbI}_{3} \mathrm{NCs}$ in the strong photoexcitation regime are investigated. In the former two NC systems, amplified spontaneous emission is found to dominate over the radiative recombination at average carrier occupancy per nanocrystal larger than 5-10. On the other hand, under the same photoexcitation conditions in the FAPbI ${ }_{3} \mathrm{NCs}$ a longer lived population of hot carriers results in a competition between hot luminescence, stimulated emission, and defect recombination. The dynamic interplay between the aforementioned three emissive channels appears to be influenced by various experimental and material parameters that include temperature, material purity, film morphology, and excitation pulse width and wavelength.

KEYWORDS: perovskites, lead halides, nanocrystals, hot carriers, amplified spontaneous emission, hot luminescence

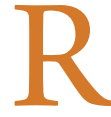

ecent work provides conclusive evidence of comparatively slow thermalization of carriers in lead halide perovskites (LHP). At low to moderate carrier concentrations, the cooling slowdown appears to originate from the screening of the carrier-optical phonon interaction promoted by the formation of polarons in the ionic LHP lattice $^{1,2}$ and/or the formation of a hot optical phonon population via the hot phonon bottleneck effect and the upconversion of low energy acoustic phonons. ${ }^{3-5}$ At high carrier concentrations, multicarrier Auger heating ${ }^{6}$ appears as the most prominent mechanism that effectively retards the dissipation of the hot carrier excess kinetic energy. ${ }^{7}$ Apart from the implications of the prolonged carrier cooling for solar cell devices, making feasible the harvesting of the hot carrier energy to challenge the Shockley-Queisser limit, ${ }^{8,9}$ the effect is expected to have a profound impact on the luminescence process that is intimately related with the energy and momentum relaxation of the excited carriers. Indeed the retarded carrier thermalization in LHPs has been shown to influence the dynamics of the spontaneous and the stimulated emission via the appearance of short-lived hot carrier photoluminescence (PL) transients and the delayed onset of optical gain. ${ }^{10,11}$ Band-filling effects resulting in blue shift and high energy broadening of the emission spectra have been demonstrated in bulk hybrid perovskite films, ${ }^{12}$ single-crystal

Received: February 19, 2019

Accepted: May 9, 2019

Published: May 9, 2019 

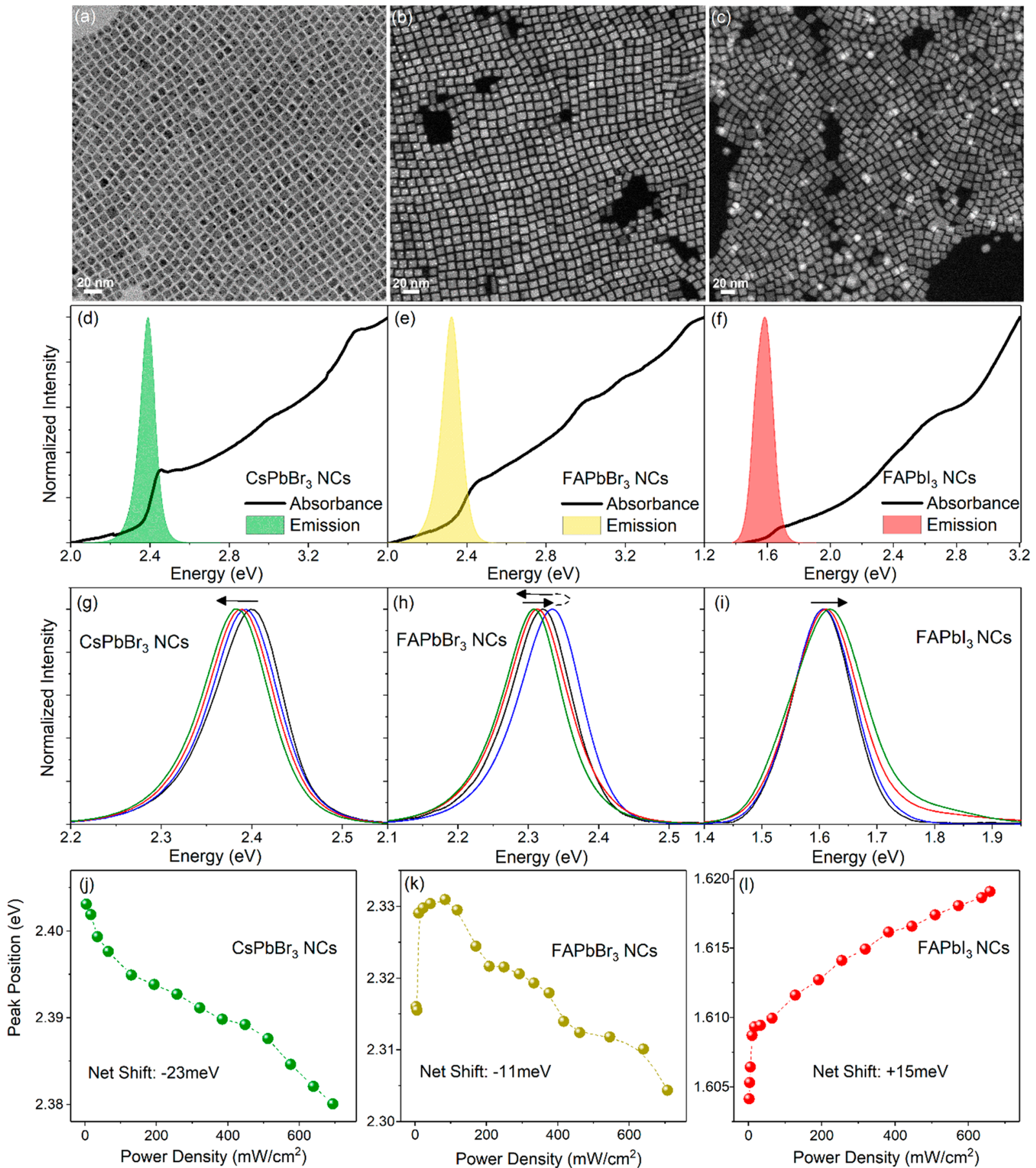

Figure 1. (a) TEM image of the CsPbBr ${ }_{3}$ NCs. Dark-field STEM images of (b) $\mathrm{FAPbBr}_{3}$ and (c) $\mathrm{FAPbI}_{3} \mathrm{NCs}$ Absorption and emission spectral from (d) $\mathrm{CsPbBr}_{3}$, (e) $\mathrm{FAPbBr}_{3}$ and (f) $\mathrm{FAPbI}_{3} \mathrm{NC}$ films. Excitation-dependent steady-state luminescence from the (g) $\mathrm{CsPbBr}_{3}$, (h) $\mathrm{FAPbBr}_{3}$, and (i) $\mathrm{FAPbI}_{3} \mathrm{NC}$ films for densities in the $1-700 \mathrm{~mW} / \mathrm{cm}^{2}$ range. The arrows indicate the respective blue/red excitation-

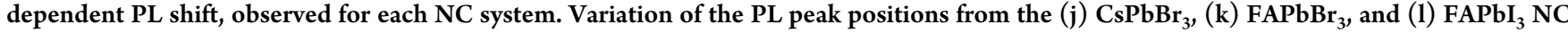
films across the aforementioned fluence range.

lead bromide perovskites, ${ }^{1}$ and tin iodide perovskite films, ${ }^{13}$ indicating that a substantial population of hot carriers can be maintained for sufficient long time scales to allow radiative recombination to occur. Such demonstrations combined with breakthrough work that proved the feasibility of efficient extraction of hot carriers via interfacial charge transfer ${ }^{14,15}$ and long-range transport ${ }^{16}$ suggest that hot carrier electronic devices based on perovskites should be feasible. Nanoscale analogues of perovskites such as colloidal nanocrystals (NCs) may bring an added benefit, as recent work indicates that the intraband carrier relaxation is further retarded compared to their bulk-crystal counterparts due to Auger heating that is efficient at systems of reduced dimensionality in the high excitation regime. ${ }^{14,17}$ LHP NCs have recently emerged as functional optical materials with outstanding light emitting and optical amplification properties, ${ }^{18-20}$ yet the influence of the prolonged carrier relaxation on the energetics and dynamics of radiative recombination under sufficiently high photon 

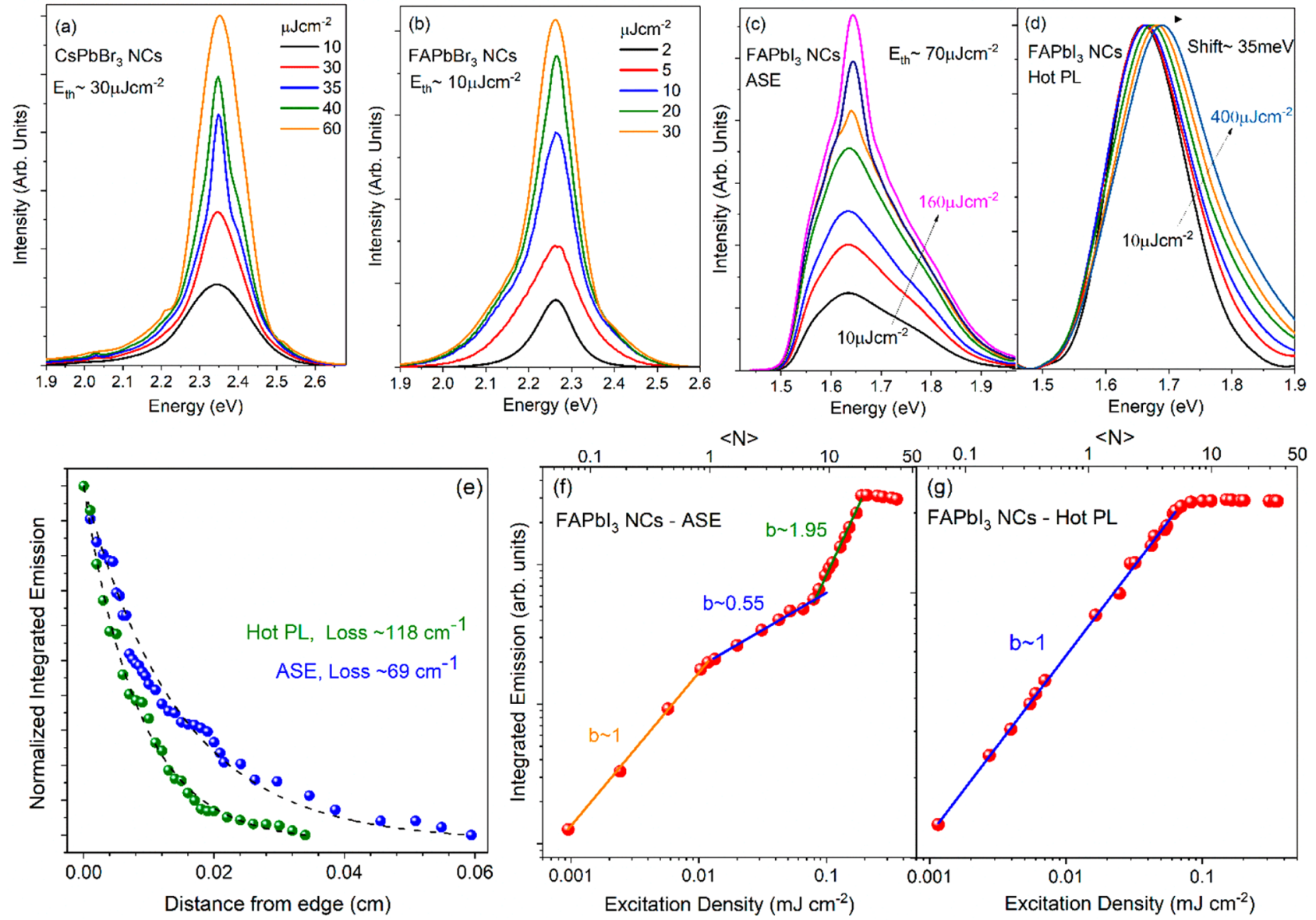

Figure 2. Excitation-dependent luminescence excited by $400 \mathrm{~nm}$ fs pulses from (a) $\mathrm{CsPbBr}_{3},(\mathrm{~b}) \mathrm{FAPbBr}_{3}$, and (c) $\mathrm{FAPbI}_{3} \mathrm{NC}_{\text {films, }}$ showing the activation of ASE. The respective ASE thresholds are also displayed. (d) fs-excited normalized emission from a FAPbI ${ }_{3} \mathrm{NC}_{\text {film }}$ where hot carrier luminescence is evidenced by the fluence-dependent blue shift and high energy broadening of the emission. (e) Results of a VSL experiment and the associated fits by a Beer-Lampert function (black dashed curves) for two films produced out of the same NC solution. The respective optical loss coefficients extracted from the Beer-Lampert fits are also displayed. Log-log plots of the integrated emission versus excitation density from $\mathrm{FAPbI}_{3} \mathrm{NC}$ films dominated by (f) ASE and (g) hot PL effects. The respective allometric fits and extracted exponents are displayed.

fluences that allow both spontaneous and stimulated emission to occur has not been explicitly and thoroughly investigated.

We present herein a comprehensive study of the luminescent properties of hot electron-hole gas in colloids and films of three representative LHP NCs based on the inorganic $\mathrm{CsPBr}_{3}$ and the hybrid $\mathrm{FAPbBr}_{3}$ and $\mathrm{FAPbI}_{3}$ materials, where $\mathrm{FA}$ stands for formamidinium. Our experiments probe the high excitation regime with average carrier occupancy per nanocrystal larger than 1, produced by excitation light pulses with temporal dynamics that span from the steady-state to the femtosecond regime. Ultrafast transient absorption spectra provide evidence of prolonged carrier cooling times in all three NC systems; however, a fluence-dependent retardation of the carrier relaxation to tens of picoseconds (ps) appears pronounced only in $\mathrm{FAPbI}_{3} \mathrm{NCs}$. Intense excitation of the three types of LHP NC materials with short pulses in the femtosecond ( $\mathrm{fs}$ ) to nanosecond (ns) time domain, results in efficient population inversion and low-threshold amplified spontaneous emission (ASE). However, in $\mathrm{FAPbI}_{3} \mathrm{NCs}$, in which the hot carrier plasma can be maintained for longer times, the carrier cooling dynamically competes with the buildup of the stimulated emission. Previous work by some of the manuscript authors ${ }^{17}$ demonstrated the presence of long- lived hot carriers in $\mathrm{FAPbI}_{3} \mathrm{NCs}$ but found no significant impact on the energetics of the NC photoluminescence for moderate excitation conditions up to $\sim 2$ carriers per nanocrystal. By extending the work to significantly higher fluences, the impact of hot carriers on the emission becomes evident when NCs are populated with more than 5 to 10 carriers, resulting in some cases in an oscillatory transient behavior and suppression of the ASE in favor of higher energy emission from hot carriers and recombination from localized defect states above the band-edge. We probe the energetics and dynamics of the aforementioned radiative channels in $\mathrm{FAPbI}_{3} \mathrm{NC}$ films and colloids and estimate average lifetimes of $\sim 0.8, \sim 0.5$, and $\sim 1.5 \mathrm{~ns}$ for the ASE, hot PL, and defect recombination, respectively. Finally we demonstrate the influence of a number of experimental and material parameters on the competition between the three mechanisms that include temperature, material purity, film quality and excitation pulse duration and wavelength. The work provides insight into hot carrier recombination that competes with the stimulated emission process affecting the photophysical and gain properties of lead halide perovskite nanocrystals. 


\section{RESULTS AND DISCUSSION}

High resolution TEM/STEM images of the three cubic-shaped LHP NC types studied are included in Figure 1a-c, while typical absorption and photoluminescence (PL) spectra from respective NC films are displayed in Figure 1d-f. All films exhibit bright PL with absolute quantum yields of $67 \%, 78 \%$, and $42 \%$ and narrow line width of ca. $\sim 75, \sim 85$, and $\sim 100$ $\mathrm{meV}$ for $\mathrm{Cs} \mathrm{PbBr}_{3}, \mathrm{FAPbBr}_{3}$, and $\mathrm{FAPbI}_{3} \mathrm{NCs}$, respectively. Increasing the excitation density up to $700 \mathrm{~mW} / \mathrm{cm}^{2}$ yields a net bathochromic shift of the luminescence in the $\mathrm{FAPbBr}_{3}$ and $\mathrm{Cs}_{\mathrm{PbBr}}$ NCs, whereas the $\mathrm{FAPbI}_{3} \mathrm{NC}$ emission consistently shifts to blue as seen in Figure $1 \mathrm{~g}-\mathrm{i}$. The absolute magnitude of the shifts in the three systems lies in the 10-25 $\mathrm{meV}$ range, and it appears to be reproducible after multiple swings of the excitation density, indicating the absence of noticeable NC photodegradation processes during the study. At higher fluences of $\sim 1 \mathrm{~W} / \mathrm{cm}^{2}$, the integrity of the NC films is compromised and an irreversible quenching of the luminescence intensity is observed in the majority of the probed films.

High-temperature PL experiments performed under intense photoexcitation up to $400 \mathrm{~K}$, presented in Supplementary Figure 1, show that heating results in monotonic blue shift of the emission from all studied films, consistent with the positive thermal expansion coefficient of the energy gap of metal halide perovskites. $^{21}$ Lattice heating by the excitation laser beam should thus be excluded as the origin of the luminescence red shift of the $\mathrm{FAPbBr}_{3}$ and $\mathrm{Cs} \mathrm{PbBr}_{3}$ NC films, apart possibly for an initial blue shift observed in the FA-based system, as seen in Figure 1h,k. The fluence-dependent bathochromic shift appears instead, consistent with bandgap renormalization and in agreement with recent work reporting on the strong manifestation of the effect in perovskite NCs. ${ }^{2,23}$ On the other hand, a different model is needed to interpret the hypsochromic emission variation in $\mathrm{FAPbI}_{3} \mathrm{NC}$ films. A possible heating contribution may account for the steep high energy shift of the luminescence at low fluences observed in Figure 11; however, the overall behavior is not consistent with sample heating as it results in an overall broadening of the PL line-shape as evidenced by the spectra in Supplemenatry Figure 1e, compared to a clearly anisotropic spread of the high energy PL branch of the fluence-dependent data of Figure 1i. The temperature-dependent data provide in addition evidence for the emergence of a second emission feature above the bandedge, the origin of which is discussed later in the manuscript. Based on the outcome of the control experiments and the appearance of the characteristic fluence-dependent, high energy tail, the hypsochromic shift in the $\mathrm{FAPbI}_{3} \mathrm{NC}$ films is predominantly attributed to a Burstein-Moss state filling of the band edge states and the radiative recombination of carriers with kinetic energies higher than the lattice temperature. The appearance of the hot luminescence even at steadystate photoexcitation conditions indicates a retardation of the hot carrier lifetimes at sufficiently long time scales to allow spontaneous emission to occur, confirming our earlier studies on the material. ${ }^{17}$

Intense photoexcitation using pulses in the $\sim 100$ fs regime typically led to the emission narrowing and activation of efficient amplified spontaneous emission (ASE), as observed in the time-integrated data of Figure 2, with thresholds as low as $\sim 30, \sim 10$, and $\sim 70 \mu \mathrm{J} \mathrm{cm}{ }^{-2}$ for $\mathrm{CsPbBr}_{3}, \mathrm{FAPbBr}_{3}$, and $\mathrm{FAPbI}_{3} \mathrm{NC}$ films, respectively. In the films of the first two material systems, in which optical losses prevail over the optical gain suppressing the ASE, a bandgap renormalizationinduced red shift of the emission is observed, in agreement with the steady-state excitation experiments. On the other hand, a different behavior is observed for $\mathrm{FAPbI}_{3} \mathrm{NCs}$, in which ASE quenching occurs at the expense of hot luminescence, evidenced by the appearance of a fluencedependent PL blue shift and the characteristic high energy broadening of the emission seen in Figure $2 \mathrm{~d}$.

The competition between optical loss and gain and its impact on the hot carrier recombination in the $\mathrm{FAPbI}_{3} \mathrm{NCs}$ is probed via an experiment producing a "good" and a "bad" film out of the same parent NC solution. The "good" film was imposed to the standard, optimized processing protocol employed for all discussed films in the manuscript and exhibits ASE upon increase of the excitation density. The "bad" film was deposited while the quartz substrate was heated to $90{ }^{\circ} \mathrm{C}$ to induce fast evaporation of the solvent and intentionally deteriorate the morphological characteristics of the film; as a result, the excitation-dependent emission yields ASE suppression at the expense of hot PL. The optical quality of the two films was quantified by the optical losses estimated using the variable stripe length (VSL) experimental method. The optical loss coefficient was extracted by Beer-Lambert type of fits as described in ref 24 and yields losses in the "bad" film increased by $\sim 70 \%$ compared to those estimated in the "good" film as shown in Figure 2e. The increased losses result in suppression of the net modal gain and weakening of the competing stimulated emission channel, activating hot carrier emission. It appears then that the local film microstructure is a determining factor, distinguishing the $\mathrm{FAPbI}_{3} \mathrm{NC}$ films into the aforementioned type of solids based on their high excitation emission properties. The result is consistent with recent microscopy studies in $\mathrm{CH}_{3} \mathrm{NH}_{3} \mathrm{PbI}_{3}$ polycrystalline films in which the critical role of film morphology on the thermalization and the carrier temperatures of hot carriers has been demonstrated. $^{25}$

The two types of $\mathrm{FAPbI}_{3} \mathrm{NC}$ films are also distinct in terms of their integrated emission excitation dependence. The first type of solids, named ASE films for the rest of this work, exhibit the typical excitation dependence observed in high gain $\mathrm{FAPbI}_{3} \mathrm{NC}$ solids ${ }^{24}$ seen in the log-log plot of Figure $2 \mathrm{f}$ with a transition from linear to sublinear behavior at a carrier occupancy of $\sim 1$, defining a respective transition from single exciton monomolecular to Auger multiexciton recombination. At higher fluences, a threshold type of behavior defines the onset of stimulated emission with the ASE threshold determined by the sub- to superlinear change of slope. Films in which emission from hot carriers prevails, coded as hot PL films, exhibit a similar monomolecular recombination at low fluences; however, no transition to the Auger sublinear dependence is observed at $\langle N\rangle \sim 1$ as seen in the ASE films. The reason for the apparent suppression of the Auger recombination is not clear; it is plausible that in the hot PL films, Auger quenching is compensated by Auger heating observed across the aforementioned fluence range in $\mathrm{FAPbI}_{3}$ NCs. ${ }^{17}$ Monomolecular recombination is accompanied by full PL saturation at higher fluences, i.e., plot of Figure $2 \mathrm{~g}$. Interestingly, the emission saturation in the hot PL films occurs within the same photoexcitation regime, i.e., average number of photoexcited carriers per nanocrystal $\langle N\rangle$ of 5-10, in which the ASE onset is found in the ASE films. It appears then that ASE and hot luminescence are competing recombination 


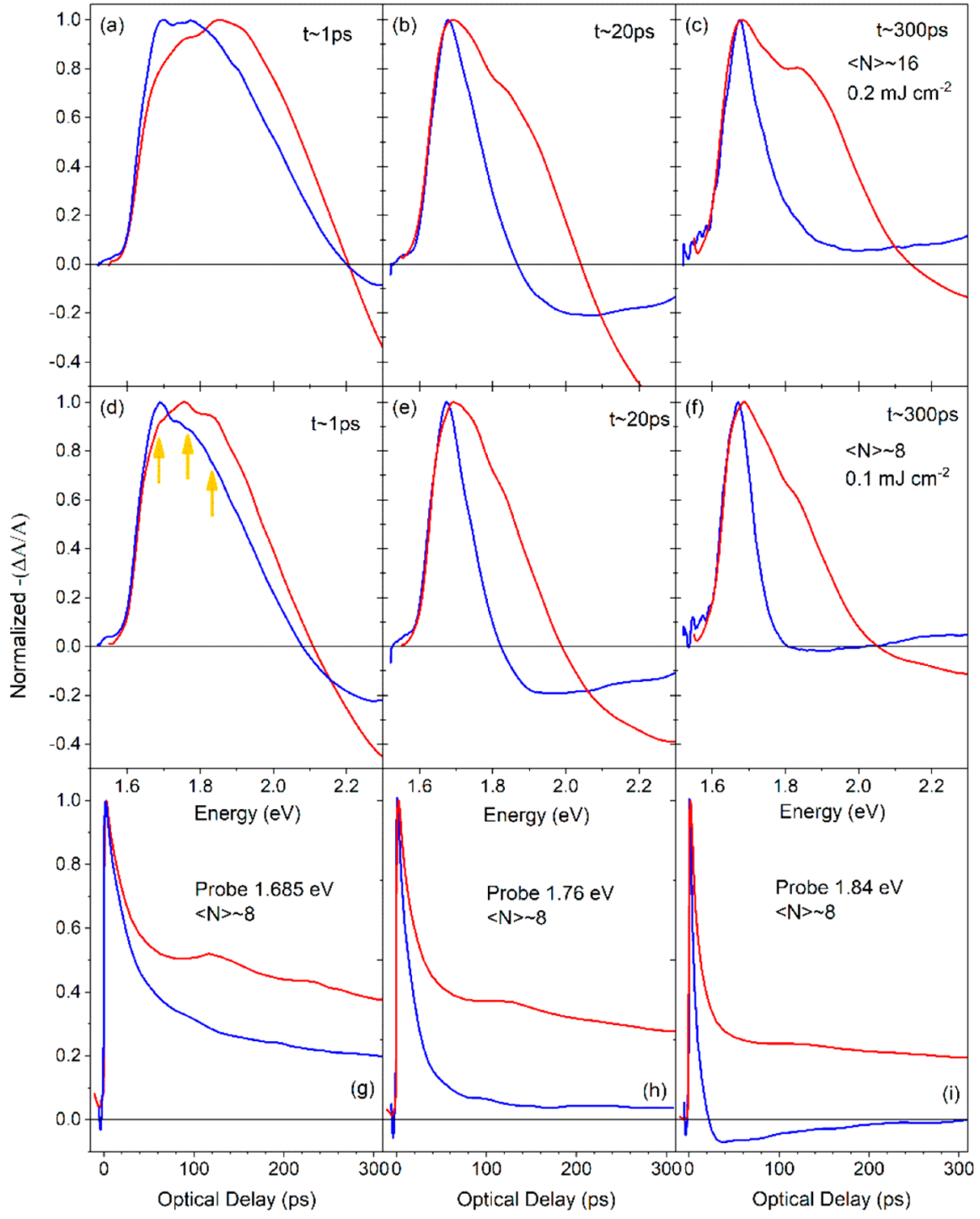

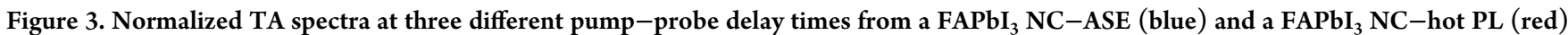
films at excitation pump fluences of $(\mathrm{a}-\mathrm{c}) 0.2 \mathrm{~mJ} \mathrm{~cm}{ }^{-2} /\langle N\rangle \sim 16$ and $(\mathrm{d}-\mathrm{f}) 0.1 \mathrm{~mJ} \mathrm{~cm}{ }^{-2} /\langle N\rangle \sim 8$. (g-i) Pump-probe transients from the two films obtained at three probe energies across the NC bleaching region indicated by the arrows in Figure $4 \mathrm{~d}$.

mechanisms in $\mathrm{FAPbI}_{3} \mathrm{NC}$ solids under high excitation powers.

Transient pump-probe absorption (TA) experiments further support the results of Figure 2. Two-dimensional TA plots and graphs of the bleaching build-up time are contained in Supplementary Figures 2 and 3, respectively, along with a short data discussion. The experiments confirm the presence of long-lived hot carriers in all three NC materials with similar dynamics at low fluences. As excitation fluence increases, significant longer cooling times are observed in the $\mathrm{FAPbI}_{3} \mathrm{NC}$ solids as a result of the longer biexciton lifetimes in such $\mathrm{NCs}^{26,27}$ compared to those observed in Cs-based $\mathrm{NCs}^{28}$ and the activation of efficient Auger heating. ${ }^{17}$

For the rest of this work, we focus on the interplay between stimulated emission and hot luminescence in $\mathrm{FAPbI}_{3} \mathrm{NCs}$, aiming to explore which other experimental and material parameters along with local film microstructure influence the dynamics and energetics of the two competitive recombination channels. Comparative pump probe spectra and transients in the TA bleaching region of the two types of films are displayed in Figure 3. Consistent with the results of Supplementary
Figure 3, both types of films feature an excitation-dependent broadening of the high-energy tail of the TA bleaching signal. However, for all cases examined, hot PL NC films exhibit a substantially longer lived and spectrally broadened TA bleaching region, with populated states extending as high as $350 \mathrm{meV}$ above the band-edge, confirming the pronounced Burstein-Moss state filling in such nanocrystals. As the spectral position of the ASE peak lies in the vicinity of the NC energy gap, the carriers must thermally relax to the band edge to contribute to the stimulated emission. It appears that the prolonged cooling and reheating of the carriers in the hot PL solids delays the onset of the population inversion, suppressing the ASE emission in favor of the competitive emission from non-thermalized carriers. The dynamic antagonism of the two processes is evident in the transients of Figure $3 g-i$ where three energies within the TA bleaching region, denoted by the arrows in Figure $3 \mathrm{~d}$, are probed.

In all cases, the ASE films exhibit faster TA dynamics to those of hot PL films. The decays are adequately described by a double exponential model with curve fits for excitation densities of $\langle N\rangle \sim 8\left(\sim 0.1 \mathrm{~mJ} \mathrm{~cm}{ }^{-2}\right)$ and $\langle N\rangle \sim 16(\sim 0.2 \mathrm{~mJ}$ 

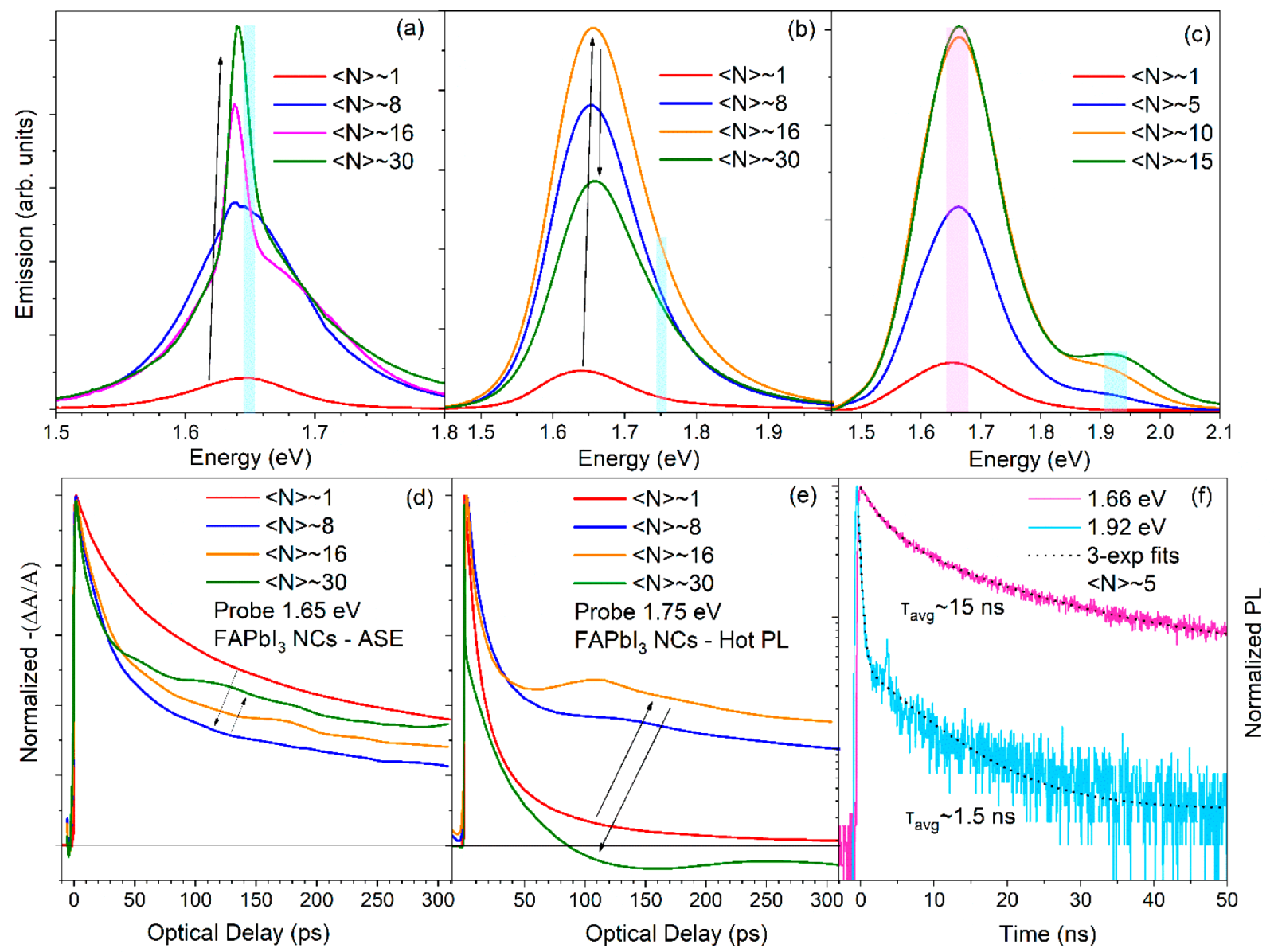

Figure 4. Excitation-dependent emission spectra from (a) $\mathrm{FAPbI}_{3} \mathrm{NC}-\mathrm{ASE}$ film, (b) $\mathrm{FAPbI}_{3} \mathrm{NC}-$ hot PL film, and (c) FAPbI $\mathrm{NC}_{3}-\mathrm{hot}_{\mathrm{PL}}$ film in which a second high energy peak emerges as excitation increases. TA decays obtained from the former two films probing the dynamics of (d) ASE emission and (e) hot PL emission in the cyan highlighted regions. The arrows indicate the sequence of TA spectra as excitation fluence increases. (f) Time-resolved PL obtained from the film studied in (c), with decays monitoring the main (red) and high energy (cyan) emission peak.

$\mathrm{cm}^{-2}$ ) displayed in Supplementary Figure 4 and fitting parameters presented in Supplementary Table 1. The model yields a short lifetime in the $\sim 8-20$ ps range with similar dynamics for both types of films, tentatively assigned to Augerassisted intraband relaxation. ${ }^{17}$ The longer $\tau_{2}$ time constant, on the other hand, lies in the $\sim 0.8-1$ ns range for the hot PL films, increased almost by a factor of 2 compared to the dynamics of the respective channel in the ASE samples. To further investigate the nature of the long decay channels in the two type of films, we performed a transient absorption study in which the photoexcited carriers per nanocrystal was varied widely in the $\langle N\rangle$ of $1-30$ range while probing within the spectral region of hot carrier luminescence and ASE emission, respectively. The results of the TA study along with the respective fluence-dependent emission spectra from the same films are displayed in Figure 4.

For the ASE film, an increase of the fluence from $\langle N\rangle \sim 1$ to $\langle N\rangle \sim 8$ yields an activation of the stimulated emission as evidenced by the emission narrowing in the steady-state regime and a concomitant quenching of the transient dynamics due to the increased radiative recombination rate. Further increase of the excitation density up to $\langle N\rangle \sim 30$ results in a modest slowing of the TA kinetics, as a larger population of hot carriers is prepared, retarding the stimulated emission build-up. The $\sim 0.5 \mathrm{~ns}$ decay channel present in the pump-probe transients for fluences above the ASE threshold is then assigned to the ASE lifetime. This interpretation is consistent with the fact that such channel quenches when probing at energies beyond the optical gain region of the NCs, i.e., at $1.84 \mathrm{eV}$ as seen in Figure $3 \mathrm{i}$ and Supplementary Figure 4c,e.

For the hot PL film of Figure $4 \mathrm{~b}$, the emission intensity increases with fluence up to $\langle N\rangle \sim 16$, while the PL peak blue shifts and asymmetrically broadens, marking the activation of hot luminescence. In the TA spectra, an identical increase in the photoexcited carrier population in the NCs results in lengthening of the TA dynamics and the appearance of the approximately ns long $\tau_{2}$ decay channel due to fluencedependent retardation of the hot carrier relaxation, also evidenced by the TA spectra of Figure 4. Further increments of the photoexcitation up to $\langle N\rangle \sim 30$ result in luminescence quenching and a concomitant shortening of the TA dynamics with a transition from bleaching to photoinduced absorption. Based on the consistent behavior of the steady-state emission and transient absorption variations with fluence, we tentatively assign the nanosecond transient as the hot luminescence decay in the $\mathrm{FAPbI}_{3}$ NCs. Similar hot PL dynamics were reported in $\mathrm{FASnI}_{3}$ films. ${ }^{13}$ On the other hand, the hot PL decay was found to be shorter lived, by an order of magnitude, in single crystals of $\mathrm{MAPbBr}_{3}$ and $\mathrm{FAPbBr}_{3}$. 

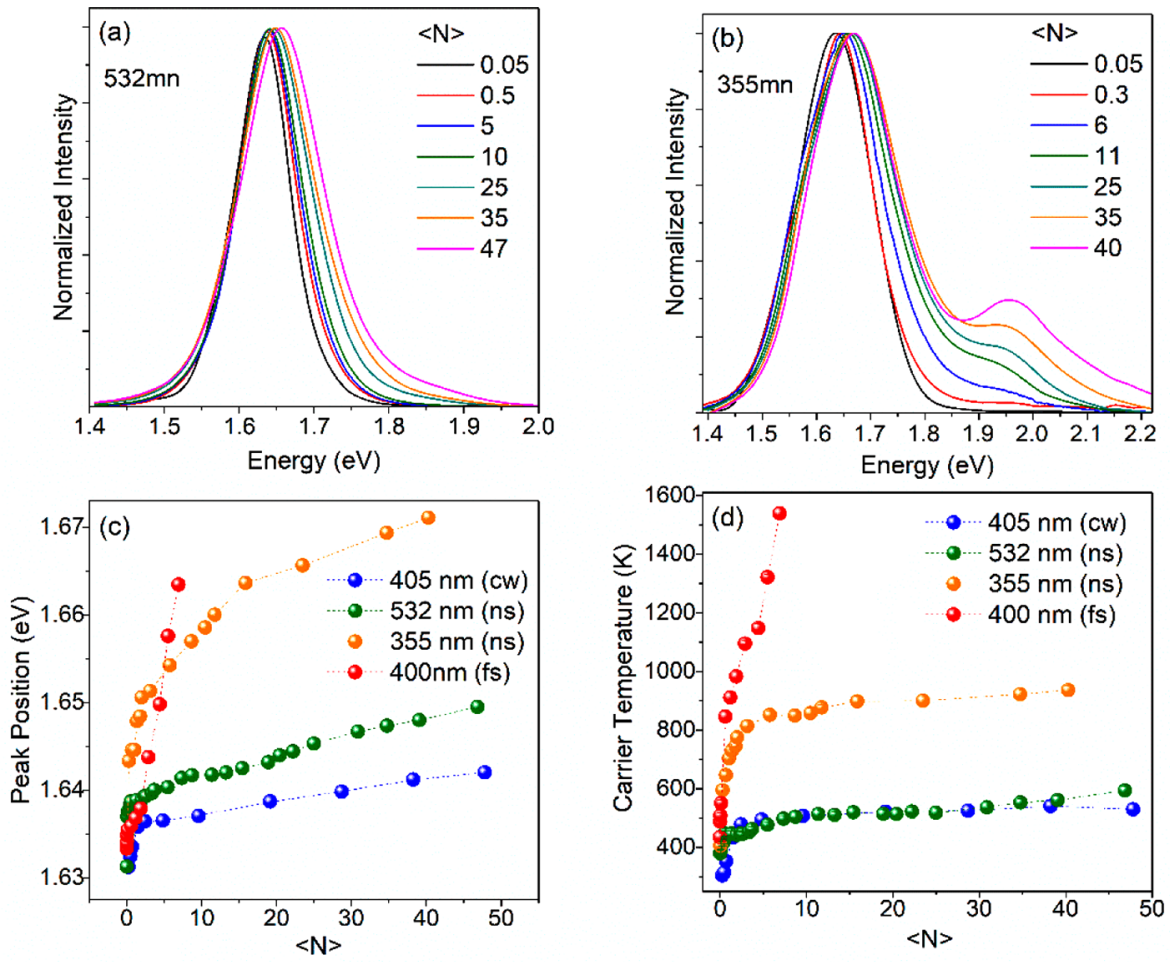

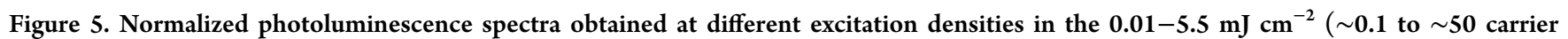
occupancy per $\mathrm{NC}$ ) range for a $\mathrm{FAPbI}_{3} \mathrm{NC}$ solution under (a) $532 \mathrm{~nm}$ ns excitation and (b) $355 \mathrm{~nm}$ ns photoexcitation. (c) Excitation dependence of the peak PL position from the aforementioned $\mathrm{FAPbI}_{3} \mathrm{NC}$ solution for different excitation pulses/wavelengths. (d) Fluence dependence of carrier temperatures $T_{c}$ extracted by the Maxwell-Boltzmann fits of eq 1 for emission obtained by different laser excitation pulses/wavelengths.

A confirmation of the dynamic competition of the two processes is the appearance of temporal oscillations of the TA signal. The oscillatory behavior is pronounced on the hot PL films transients at probes in the vicinity of the NC band-edge, where oscillations with a period of $\sim 110-130 \mathrm{ps}$ are visible, as seen in Figure $3 \mathrm{~g}$ and Figure 4c. Oscillations have also been reported in the time-resolved luminescence transients of bulk hybrid perovskite crystals ${ }^{10}$ and appear to originate on cycles of depletion of the NCs ground state via ASE followed by ASE regeneration as long-lived hot carriers relax to the bottom of the band. In our experiments, the oscillatory behavior exhibits a period larger by an order of magnitude compared to the $\sim 10$ ps oscillations reported in the work above, which appears consistent with the significantly prolonged hot carrier lifetime in nanoscale analogs of hybrid perovskites. 14,17

In a fraction of the hot PL films, a second emission feature, at energies of $200-300 \mathrm{meV}$ above the $\mathrm{FAPbI}_{3}$ band-edge emission, emerges at high photoexcitation or high sample temperature as seen in Figure $4 c$ and Supplementary Figure 1e, respectively. The transient PL data of Figure $4 \mathrm{f}$, yields an average PL lifetime of $\sim 1.5 \mathrm{~ns}$ for the high energy peak, quenched by an order of magnitude compared to the dynamics of the main NC emission. A similar short-lived, emission feature above the band-edge was reported recently in the high excitation $\mathrm{PL}$ spectra of $\mathrm{MAPbB}_{3} \mathrm{NCs}$ and has been assigned to radiative recombination from higher electronic NC states. ${ }^{29}$ In our work, the feature is tentatively assigned to a localized defect state, supported by the findings of a differential excitation PL (PLE) experiment discussed in Supplementary Figure 5. It is noted that the formation of the band gap via sets of antibonding orbitals in perovskite crystals allows the formation of such localized trap states that reside in energies within the valence and conduction band of the material. ${ }^{18}$ The experimental and material characteristics that diversify the studied $\mathrm{FAPbI}_{3} \mathrm{NCs}$ into the two distinct types are further investigated via a parametric photoluminescence study. We first discuss the studies on $\mathrm{FAPbI}_{3} \mathrm{NC}$ colloids with the experimental summary reported in Figure 5.

The hot luminescence was quantified by the magnitude of the fluence-dependent Burstein-Moss emission blue shift and the high energy tail broadening. The tail emission due to hot carriers with energies substantially larger than the quasi-Fermi $E_{\mathrm{F}}$ level can be fitted by a Maxwell-Boltzmann function ${ }^{30}$ of the form

$$
I(E)=I_{0} \cdot \mathrm{e}^{-E / k_{\mathrm{B}} T_{c}}
$$

with $I_{0}$ as the PL tail point in which the emission intensity assumes half of its maximum value $I_{\max }$ i.e., $I_{0}=0.5 I_{\max }$, and the fitting parameter as the carrier temperature $T_{c}$. The model produces good fits of the data for NC emission excited via $\sim 100$ fs pulses, $5 \mathrm{~ns}$ pulses, and continuous wave laser excitation, as observed in Supplementary Figure 6, yielding for each experiment the hot carrier temperature values $T_{c}$. On the basis of the extracted carrier temperatures and the emission blue shift the impact of photoexcitation wavelength and pulse duration on the hot luminescence characteristics of the $\mathrm{FAPbI}_{3}$ NC colloids was evaluated; in addition, the impact of purification steps during the NC processing was analyzed, and the respective data summary is displayed in Supplementary Figure 7. Pumping the NC colloids at higher excitation energy, i.e., with a UV versus a green laser, while keeping the excitation pulse width fixed at $\sim 5 \mathrm{~ns}$ results in the generation of carriers 

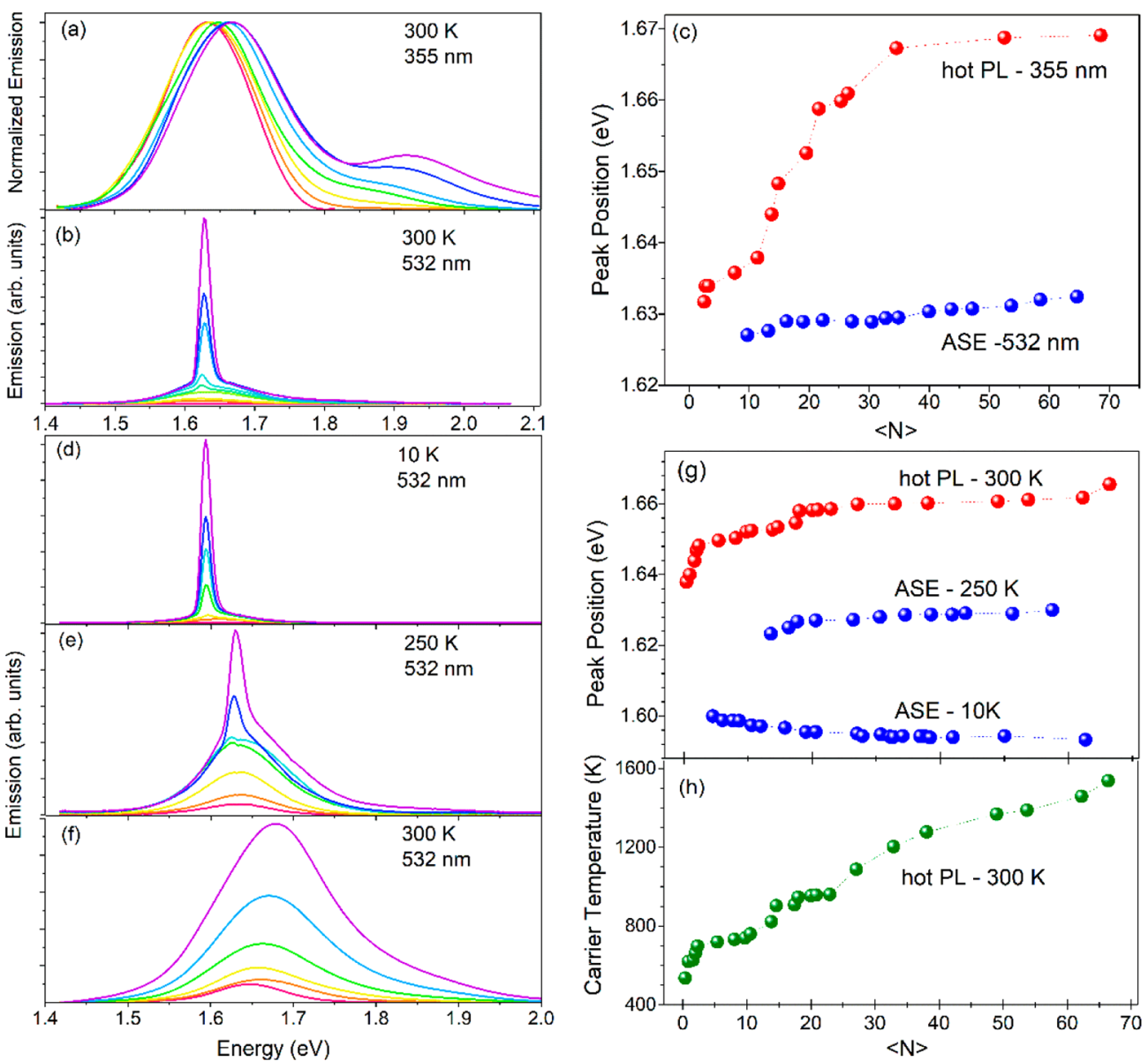

Figure 6. Fluence-dependent PL spectra from a $\mathrm{FAPbI}_{3} \mathrm{NC}$ film excited by nanosecond pulses with a wavelength of (a) $355 \mathrm{~nm}$, (b) $532 \mathrm{~nm}$. (c) Emission peak versus excitation density for the experiments above. Photoluminescence from a $\mathrm{FAPbI}_{3} \mathrm{NC}$ film at (d) $10 \mathrm{~K}$, (e) $250 \mathrm{~K}$, and (f) $300 \mathrm{~K}$, excited by $532 \mathrm{~nm}$ nanosecond pulses. (g) Variation of the emission peak versus excitation fluence for the three sample temperatures. (h) Extracted carrier temperatures versus average carrier population per nanocrystal for the $\mathrm{FAPbI}_{3} \mathrm{NC}_{\mathrm{emission}}$ spectra at 300 K.

with larger kinetic excess energy, increased $T_{\mathcal{c}}$, and larger hot emission contribution as evidenced by the data of Figure 5. UV excitation typically excites the high energy defect luminescence peak as well as seen in Figure 5b. On the other hand, the increase of the excitation pulse width results in a dynamic competition of the hot luminescence with quenching mechanisms such as hot carrier relaxation and nonradiative recombination processes, effectively reducing the effect as seen in Figure 5c,d. The photoexcitation wavelength exhibits an even more pronounced impact on the radiative recombination of hot carriers in films of $\mathrm{FAPbI}_{3} \mathrm{NCs}$. Upon varying the excitation wavelength from green to UV, the stimulated emission can be fully suppressed in favor of hot luminescence, as seen in Figure $6 \mathrm{a}-\mathrm{c}$; the stimulated emission can be reactivated upon switching back to green illumination of the NC film. The interplay of the two recombination mechanisms can also be controlled by the variation of the sample temperature. For the example illustrated in Figure $6 \mathrm{~d}-\mathrm{h}$, sample heating from 10 to $250 \mathrm{~K}$ results in continuous increase of the ASE threshold while the bandgap renormalizationinduced, excitation-dependent red shift of the PL and ASE peak at $10 \mathrm{~K}$ is replaced by a Moss-Burstein blue shift of the spontaneous and stimulated emission at $250 \mathrm{~K}$. Further heating up to $300 \mathrm{~K}$ results in complete quenching of the optical amplification and the appearance of significant emission from nonthermalized carriers. Overall, an upper bound in carrier temperatures of $\sim 1600 \mathrm{~K}$ was obtained for both $\mathrm{FAPbI}_{3} \mathrm{NC}$ films and colloids in our studies, closely matching respected carrier temperatures measured in the hot PL experiments of bulk films of tin-iodide perovskites ${ }^{13}$ and single crystals of $\mathrm{MAPbBr}_{3}$ and $\mathrm{FAPbBr}_{3}$.

An interesting observation related to the dynamics of the carrier band filling is that for low to moderate fluences up to $\langle N\rangle \sim 2$, the high energy tail of the transient absorption spectra in $\mathrm{FAPbI}_{3} \mathrm{NC}$ films and colloids were found to be well approximated by a Maxwell-Boltzmann type of function. ${ }^{17} \mathrm{On}$ the other hand, for higher fluences, the TA spectra assume a complex nonexponential high energy tail shape (see Figure 3) that cannot be fitted by the aforementioned function. It appears that intense photoexcitation activates many-body interactions that inhibit the fast formation of a Fermi-Dirac type of carrier distribution. Nonetheless, in all hot luminescence experiments, the formation of a well-shaped exponential emission tail is consistently observed. The discrepancy may be justified by taking into consideration that transient absorption probes the dynamics of all photoexcited carriers while luminescence is sensitive only to the fraction of photogenerated carriers participating in the radiative recombination process. We hypothesize that within the relatively slow nanosecond time scale that the hot 
luminescence decays, the formation of a quasi-equilibrium of the carriers that contribute to the radiative recombination occurs, justifying the approximation of the nonthermalized emissive carriers with an exponential Maxwell-Boltzmann type of function.

The summary of the discussed work for the $\mathrm{FAPbI}_{3} \mathrm{NCs}$ is contained in the schematic model of Figure 7. The schematic

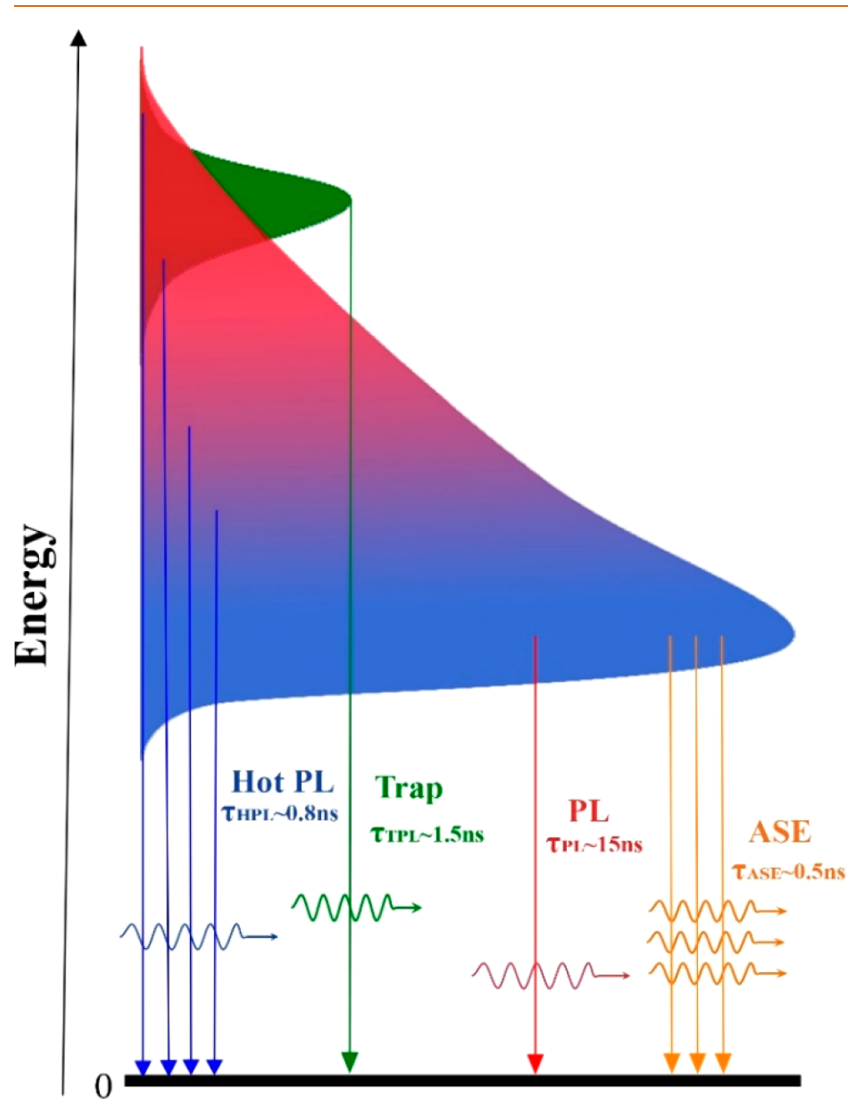

Figure 7. Model and dynamics of the recombination mechanisms of hot and cold carriers in $\mathrm{FAPbI}_{3} \mathrm{NCs}$ under intense photoexcitation conditions.

displays the competing radiative pathways of cold and hot carriers, and the respected recombination dynamics in $\mathrm{FAPbI}_{3}$ NCs observed in the high excitation regime where carrier occupancy per nanocrystal is quite larger than 1 .

\section{CONCLUSIONS}

An investigation of the radiative recombination of carriers in colloids and films of $\mathrm{CsPbBr}_{3}, \mathrm{FAPbBr}_{3}$, and $\mathrm{FAPbI}_{3} \mathrm{NCs}$, under intense photoexcitation conditions, has been performed. Recombination in the former two systems is dominated by amplified spontaneous emission; in the $\mathrm{FAPbI}_{3}$ system, we find that a long-lived population of hot carriers imparts the dynamics and energetics of the NC emission, yielding a complex competition between luminescence from nonthermalized carriers, stimulated emission and recombination from localized defect states. We probe the dynamics, explore the interplay of the three emissive channels and find dependencies with experimental and material parameters such as temperature, excitation pulse width and wavelength, material purity and film microstructure. Overall, the reported work demonstrates the profound impact of hot electron-hole gases on the spontaneous and stimulated emission process in
LHP NCs and has direct implications to the design and realization of hot solar cell absorbers and lasers based on such nanomaterials.

\section{MATERIALS AND METHODS}

Synthesis of $\mathrm{CsPbBr}_{3}$ Nanocrystals. Preparation of Cesium Oleate. Cesium carbonate (0.815g, Aldrich, $99.9 \%)$ was loaded into $100 \mathrm{~mL}$ three-neck flask along with octadecene $(40 \mathrm{~mL}$, SigmaAldrich, 90\%) and oleic acid (2.5 mL, OAc, Sigma-Aldrich, 90\%), dried for $1 \mathrm{~h}$ at $100{ }^{\circ} \mathrm{C}$, and then heated under $\mathrm{N}_{2}$ to $120^{\circ} \mathrm{C}$ until all $\mathrm{Cs}_{2} \mathrm{CO}_{3}$ reacted with OAc. Since Cs oleate precipitates out of ODE at room temperature, it has to be preheated to ca. $100{ }^{\circ} \mathrm{C}$ before injection.

Synthesis of $\mathrm{Cs} P b B r 3$ Nanocrystals. In a $25 \mathrm{~mL}$ three-necked flask, $\mathrm{PbBr}_{2}$ (69 mg, $\left.0.188 \mathrm{mmol}, \mathrm{ABCR}\right)$ was suspended in octadecene (5 $\mathrm{mL}$, ODE, Sigma-Aldrich), dried at $100{ }^{\circ} \mathrm{C}$ for $30 \mathrm{~min}$, and mixed with oleic acid $\left(0.5 \mathrm{~mL}, \mathrm{OAc}\right.$, Sigma-Aldrich, vacuum-dried at $100{ }^{\circ} \mathrm{C}$, stored in glovebox) and oleylamine (0.5 mL, OAm, STREM, vacuumdried at $100{ }^{\circ} \mathrm{C}$, stored in glovebox). When $\mathrm{PbBr}_{2}$ was dissolved, the reaction mixture was heated to $180{ }^{\circ} \mathrm{C}$, and preheated cesium oleate in octadecene $(0.4 \mathrm{~mL})$ was injected. The reaction mixture was cooled immediately with an ice bath to room temperature.

Purification and Size-Selection of $\mathrm{CsPbBr}_{3}$ Nanocrystals. The crude solution was centrifuged at $12.1 \mathrm{krpm}$ (Centrifuge: Eppendorf 5804) for $5 \mathrm{~min}$, following which the supernatant was discarded, and the precipitate was dissolved in $300 \mu \mathrm{L}$ of hexane. The hexane solution was centrifuged again, and the precipitate was discarded. The supernatant was diluted two times and used for further DDAB treatment.

$D D A B$ Treatment of $\mathrm{Cs} \mathrm{PbBr}_{3}$ Nanocrystals. Toluene $(0.6 \mathrm{~mL})$ was added to $\mathrm{CsPBr}_{3}$ colloidal solution prepared as described above. Then $30 \mu \mathrm{L}$ of $\mathrm{OAc}$ and $160 \mu \mathrm{L}$ of DDAB (didodecyldimethylammonium bromide, $0.05 \mathrm{M}$ in toluene) were added to the colloidal solution of $\mathrm{CsPBBr}_{3}$ nanocrystals. The mixture was stirred for $1 \mathrm{~h}$, followed by the precipitation with $1.8 \mathrm{~mL}$ of ethyl acetate and centrifuged at $12.1 \mathrm{krpm}$ for $3 \mathrm{~min}$. Precipitate was redispersed in 0.5 $\mathrm{mL}$ of toluene, and this solution was additionally filtered through a $0.45 \mu \mathrm{m}$ PTFE-filter.

Synthesis of $\mathrm{FAPbBr}_{3}$ Nanocrystals. Preparation of Oleylammonium Bromide (OAmBr). Oleylamine (12.5 mL, Acros Organics, $80-90 \%)$ and ethanol $(100 \mathrm{~mL}$, Aldrich) were mixed in a $250 \mathrm{~mL}$ flask. The reaction mixture was cooled in an ice-water bath, and 8.56 $\mathrm{mL} \mathrm{HBr}$ (48\% aqueous solution, Aldrich) was added. The reaction mixture was allowed to react overnight under nitrogen flow. Then the solution was dried in a rotary evaporator, the obtained product was washed multiple times with diethyl ether, and then a white powder was dried under vacuum at room temperature for several hours. $\mathrm{OAmBr}$ was stored in the glovebox.

Synthesis of $\mathrm{FAPbBr}_{3}$ Nanocrystals. In a $25 \mathrm{~mL}$ three-necked flask, lead(II) acetate trihydrate $(76 \mathrm{mg}, 0.2 \mathrm{mmol}$, Sigma-Aldrich) and formamidinium acetate $(78 \mathrm{mg}, 0.75 \mathrm{mmol}$, Sigma) were suspended in octadecene $(8 \mathrm{~mL})$ and $\mathrm{OAc}(2 \mathrm{~mL}$, Sigma-Aldrich $)$, heated to 50 ${ }^{\circ} \mathrm{C}$, and dried under vacuum for $30 \mathrm{~min}$. Then the reaction mixture was heated to $130^{\circ} \mathrm{C}$, and at this point, the mixture of $266 \mathrm{mg}(0.8$ $\mathrm{mmol}$ ) of $\mathrm{OAmBr}$ in anhydrous toluene $(2 \mathrm{~mL})$ was injected into the reaction flask (to dissolve $\mathrm{OAmBr}$ in toluene, mixture of them should be preheated at $40-50{ }^{\circ} \mathrm{C}$ ). After another $1 \mathrm{~min}$, the reaction mixture was cooled in an ice-water bath.

Purification of $\mathrm{FAPbBr}_{3}$ Nanocrystals. Methyl acetate $(16 \mathrm{~mL})$ (ABCR) was added to the crude solution followed by centrifugation at $12.1 \mathrm{krpm}$ for $5 \mathrm{~min}$ (Centrifuge: Eppendorf 5804), and the supernatant was discarded. The precipitate was dissolved in toluene $(5 \mathrm{~mL})$, and the solution was centrifuged again $(3 \mathrm{krpm}, 2 \mathrm{~min})$. The supernatant, containing monodisperse nanocrystals, was retained for the DDAB treatment, while the precipitated NCs were discarded.

$D D A B$ Treatment of FAPbBr ${ }_{3}$ Nanocrystals. Toluene $(5 \mathrm{~mL})$ was added to the $\mathrm{FAPbBr}_{3}$ colloidal solution prepared as described above. Then $0.1 \mathrm{~mL}$ of oleic acid and $0.6 \mathrm{~mL}$ of DDAB (didodecyldimethylammonium bromide, $0.05 \mathrm{M}$ in toluene) were added to $10 \mathrm{~mL}$ of 
the colloidal solution of $\mathrm{FAPbBr}_{3}$ NCs. The mixture was stirred for 1 $\mathrm{h}$ followed by precipitation with $16 \mathrm{~mL}$ of ethyl acetate, and the solution was centrifuged at $12.1 \mathrm{krpm}$ for $3 \mathrm{~min}$. The precipitate was redispersed in $5 \mathrm{~mL}$ of toluene, and this solution was additionally filtered through a $0.45 \mu \mathrm{m}$ PTFE-filter.

Synthesis of $\mathrm{FAPbl}_{3}$ Nanocrystals. Preparation of Formamidinium Oleate. Formamidine acetate $(3.765 \mathrm{mmol}, 0.392 \mathrm{~g}$, Aldrich, $99 \%$ ) was loaded into a $50 \mathrm{~mL}$ three-neck flask along with octadecene $(18 \mathrm{~mL})$ and OAc (12 mL, Sigma-Aldrich, 90\%). The reaction mixture was degassed three times at room temperature, heated to 120 ${ }^{\circ} \mathrm{C}$ under nitrogen until the reaction completed, and then cooled to room temperature. The solution was stored in a glovebox.

Synthesis of $\mathrm{FaPbl}_{3} \mathrm{NCs}$. In a $25 \mathrm{~mL}$ three-necked flask was suspended lead(II) iodide (61 mg, $0.133 \mathrm{mmol}$, Sigma-Aldrich) in octadecene $(4.6 \mathrm{~mL})$, and the mixture was heated to $60{ }^{\circ} \mathrm{C}$ and dried under vacuum for $20 \mathrm{~min}$. The reaction mixture was then heated to $110^{\circ} \mathrm{C}$ under nitrogen, and dried OAm $(0.5 \mathrm{~mL}$, STREM $)$ and dried $\mathrm{OAc}(1.0 \mathrm{~mL})$ were injected. Once the lead(II) iodide dissolved, the reaction mixture was cooled to $80{ }^{\circ} \mathrm{C}$, the mixture of formamidinium oleate $(5.0 \mathrm{~mL})$ and octadecene $(1.0 \mathrm{~mL})$ was injected, and $15 \mathrm{~s}$ later, the reaction mixture was cooled by a water bath.

Purification of $\mathrm{FAPbl}_{3}$ Nanocrystals. The crude solution was centrifuged at $12.1 \mathrm{krpm}$ for $5 \mathrm{~min}$ (Centrifuge: Eppendorf 5804), and the supernatant was discarded. The precipitate was dissolved in hexane $(0.3 \mathrm{~mL})$, and the solution was centrifuged again at $12.1 \mathrm{krpm}$ for $5 \mathrm{~min}$. Supernatant and precipitate were separated, and $0.2 \mathrm{~mL}$ of hexane was added to the supernatant. This fraction was labeled as "SN". Precipitate was dissolved $1 \mathrm{~mL}$ of toluene and centrifuged at 3 $\mathrm{krpm}$ for $2 \mathrm{~min}$ to get rid of large NCs. The result sample was labeled as "P". NCs from both fractions were washed again to remove excess of organic ligands.

(i) Purification of $\mathrm{FAPbI}_{3} \mathrm{SN}$ NCs. To $0.2 \mathrm{~mL}$ of hexane solution of NCs were added hexane $(0.2 \mathrm{~mL})$, toluene $(0.4 \mathrm{~mL})$, and methyl acetate $(0.86 \mathrm{~mL})$. The solution was centrifuged at $13.4 \mathrm{krpm}$ for 3 $\mathrm{min}$, and the precipitate was dissolved in toluene $(\sim 10 \mathrm{mg} / \mathrm{mL})$ and filtered through a $0.45 \mathrm{~mm}$ PTFE filter.

(ii) Purification of $\mathrm{FAPbI}_{3} \mathrm{P}$ NCs. A $0.65 \mathrm{~mL}$ portion of methyl acetate was added to a toluene solution of fraction " $\mathrm{P}$ " and centrifuged at $10 \mathrm{krpm}$ for $3 \mathrm{~min}$. The obtained precipitate was dissolved in toluene filtered through a $0.45 \mathrm{~mm}$ PTFE filter.

Deposition of Nanocrystal Films. Concentrated NCs solution $(20-30 \mu \mathrm{L}, 30 \mathrm{mg} / \mathrm{mL})$ was drop-casted on optical grade quartz substrates precleaned with piranha solution and pretreated with octyltriethoxysilane (96\%). Subsequent to deposition, all films were imposed to thermal annealing at $90{ }^{\circ} \mathrm{C}$ for 30 min under ambient conditions, resulting in uniform films with thicknesses in the $\sim 200-$ $300 \mathrm{~nm}$ range.

Transmission Electron Microscopy. TEM/STEM images were captured using a JEOL JEM-2200FS microscope operated at $200 \mathrm{kV}$.

Optical Spectroscopy. Absorbance of films was recorded using a PerkinElmer Lamda 1050 spectrophotometer. Continuous-wave (cw) or nanosecond excited photoluminescence (PL) and amplified spontaneous emission (ASE) experiments were carried on a $0.75 \mathrm{~m}$ Acton750i Princeton spectrometer equipped with a $1024 \times 256$ pixels PIXIS charge-coupled device (CCD) camera. The films were excited either by a cw-diode laser at $405 \mathrm{~nm}$ in the $2-1000 \mathrm{~mW} \mathrm{~cm}^{-2}$ range or via the triple, i.e., $355 \mathrm{~nm}$ or double, i.e., $532 \mathrm{~nm}$ harmonic of a Quantel Brilliant Nd:YAG laser with pulse width of $\sim 5 \mathrm{~ns}$, repetition rate of $10 \mathrm{~Hz}$, and energy density in the $0.01-12 \mathrm{~mJ} \mathrm{~cm}^{-2}$ range. Femtosecond excitation of ASE was provided by the $400 \mathrm{~nm}$ output of a Ti:sapphire ultrafast amplifier running at $1 \mathrm{kHz}$ and producing pulses of $100 \mathrm{fs}$ width. The emission was detected in such cases by a scanning spectrometer equipped with a visible photomultiplier tube. ASE and PL emission were detected using a backscattering (round excitation spot) geometry in all experiments apart from the data reported in Figure 1f,g that were acquired using the variable stripe length (VSL) method. Photoluminescence quantum yield (PL QY) was measured according to the method described in ref 31 implementing a $4 \mathrm{in}$. integrating sphere coupled to the aforementioned Princeton spectrometer via an optical fiber bundle. Temper- ature-dependent PL/ASE measurements in the $10-300 \mathrm{~K}$ range were carried out by placing the samples in the coldfinger of a Janis CCS150 closed-cycle refrigerator. Excitation PL (PLE) experiments were carried out on a FluoroLog FL3 Horiba Jobin Yvon spectrofluorimeter using the monochromator-filtered output of a $450 \mathrm{~W}$ ozone-free Xe lamp. Differential PLE experiments were performed by exciting the samples via the Xe lamp beam in the presence i.e. PLE(Xe+Nd:YAG) and absence, i.e., $\mathrm{PLE}(\mathrm{Xe})$ of a $532 \mathrm{~nm}$ beam from a Quantel Brilliant Nd:YAG laser. The differential PLE signal was then defined as

$$
\text { differential PLE }=\frac{\text { PLE }(\mathrm{Xe}+\mathrm{Nd}: Y A G)-\operatorname{PLE}(\mathrm{Xe})}{\operatorname{PLE}(\mathrm{Xe})}
$$

Time-resolved photoluminescence (TR-PL) was performed on the FluoroLog FL3 spectrofluorimeter using a time-correlated single photon counting (TCSPC) method and excited by a $375 \mathrm{~nm}$ NanoLED laser diode operating at $100 \mathrm{kHz}$.

Transient absorption measurements were carried out using the same Ti:sapphire ultrafast amplifier employed for the excitation of fsASE experiments. The system produced $1.5 \mathrm{~mJ}$ of energy per pulse and was utilized to generate pulses at $400 \mathrm{~nm}$ in a typical second harmonic configuration with a BBO crystal. A combination of a half waveplate and a polarizer were used to control the energy incident on the sample. The pump-probe setup was employed in a typical noncollinear configuration where the excitation beam at $400 \mathrm{~nm}$ was directed onto the sample at nearly normal incidence. The probe beam was incident at approximately $10^{\circ}$ to the normal and was generated using $\sim 3 \mu \mathrm{J}$ of the amplified ultrafast pulse which was focused on a 1 $\mathrm{mm}$ thick sapphire plate. Transient absorption measurements were taken using two methodologies. In the first method, lock-in amplifiers were utilized along with band-pass filters to select the probing wavelength. In this technique, transient reflection and transmission measurements were taken simultaneously at each probing wavelength. In the second technique, a fast linear array coupled to a spectrometer was utilized combined with a synchronized optical chopper at the amplifier repetition rate $(1 \mathrm{kHz})$ running at $500 \mathrm{~Hz}$, thereby achieving sensitivity comparable to that of lock-in amplifiers. The spectrometer technique allowed the simultaneous collection of transient data over a broad spectral range. For all spectroscopic work, the estimation of the average carrier occupancy per nanocrystal $\langle N\rangle$ was performed as described in previous work; see ref 17 .

\section{ASSOCIATED CONTENT}

\section{Supporting Information}

The Supporting Information is available free of charge on the ACS Publications website at DOI: 10.1021/acsnano.9b01398.

Table with transient absorption fitting parameters, hightemperature PL spectra, transient absorption contour plots, transient absorption decays and fits, PL and PLE spectra, Maxwell-Boltzmann fits of hot PL spectra, and effect of NC purification on the luminescence spectra of NC colloids (PDF)

\section{AUTHOR INFORMATION}

\section{Corresponding Author}

*E-mail: itskos@ucy.ac.cy.

ORCID $\odot$

Maryna I. Bodnarchuk: 0000-0001-6597-3266

Maksym V. Kovalenko: 0000-0002-6396-8938

Grigorios Itskos: 0000-0003-3971-3801

Notes

The authors declare no competing financial interest.

\section{ACKNOWLEDGMENTS}

This work was financially supported by the Research Promotion Foundation of Cyprus under the "NEW STRATE- 
GIC INFRASTRUCTURE UNITS-YOUNG SCIENTISTS” Programme (Grant Agreement No. "INFRASTRUCTURES/ 1216/0004", Acronym "NANOSONICS”).

\section{REFERENCES}

(1) Zhu, H.; Miyata, K.; Fu, Y.; Wang, J.; Joshi, P. P.; Niesner, D.; Williams, K. W.; Jin, S.; Zhu, X. Y. Screening in Crystalline Liquids Protects Energetic Carriers in Hybrid Perovskites. Science 2016, 353, 1409-1413.

(2) Bakulin, A. A.; Selig, O.; Bakker, J. H.; Rezus, L.A. Y.; Müller, C.; Glaser, T.; Lovrincic, R.; Sun, Z.; Chen, Z.; Walsh, A.; Frost, M. J.; Jansen, L. C. T. Real-Time Observation of Organic Cation Reorientation in Methylammonium Lead Iodide Perovskites. J. Phys. Chem. Lett. 2015, 6, 3663-3669.

(3) Yang, J.; Wen, X.; Xia, H.; Sheng, R.; Ma, Q.; Kim, J.; Tapping, P.; Harada, T.; Kee, T. W.; Huang, F.; Cheng, Y. B.; Green, M.; HoBaillie, A.; Huang, S.; Shrestha, S.; Patterson, R.; Conibeer, G. Acoustic-Optical Phonon Up-Conversion and Hot-Phonon Bottleneck in Lead-Halide Perovskites. Nat. Commun. 2017, 8, 14120.

(4) Price, M. B.; Butkus, J.; Jellicoe, T. C.; Sadhanala, A.; Briane, A.; Halpert, J. E.; Broch, K.; Hodgkiss, J. M.; Friend, R.; Deschler, F. HotCarrier Cooling and Photoinduced Refractive Index Changes in Organic-Inorganic Lead Halide Perovskites. Nat. Commun. 2015, 6, 8420.

(5) Yang, Y.; Ostrowski, D. P.; France, R. M.; Zhu, K.; van de Lagemaat, J.; Luther, J. M.; Beard, M. C. Observation of a HotPhonon Bottleneck in Lead-Iodide Perovskites. Nat. Photonics 2016, $10,53-59$.

(6) Downer, M.; Shank, C. Ultrafast Heating of Silicon on Sapphire by Femtosecond Optical Pulses. Phys. Rev. Lett. 1986, 56, 761-764.

(7) Fu, J.; Xu, Q.; Han, G.; Wu, B.; Huan, C. H. A.; Leek, M. L.; Sum, T. C. Hot Carrier Cooling Mechanisms in Halide Perovskites. Nat. Commun. 2017, 8, 1300.

(8) Shah, J. Hot Carriers in Semiconductor Nanostructures, Physics and Applications. Academic Press Inc. 1992, 279-307.

(9) Kahmann, S.; Loi, M. A. Hot Carrier Solar Cells and the Potential of Perovskites for Breaking the Shockley-QueisserLimit. J. Mater. Chem. C 2019, 7, 2471-2486.

(10) Chen, K.; Barker, A. J.; Morgan, F. L. C.; Halpert, J. E.; Hodgkiss, J. M. Effect of Carrier Thermalization Dynamics on Light Emission and Amplification in Organometal Halide Perovskites. J. Phys. Chem. Lett. 2015, 6, 153-158.

(11) Geiregat, P.; Maes, J.; Chen, K.; Drijvers, E.; De Roo, J.; Hodgkiss, M. J.; Hens, Z. Using Bulk-Like Nanocrystals to Probe Intrinsic Optical Gain Characteristics of Inorganic Lead Halide Perovskites. ACS Nano 2018, 12, 10178-10188.

(12) Dar, M. I.; Jacopin, G.; Meloni, S.; Mattoni, A.; Arora, N.; Boziki, A.; Zakeeruddin, M. Z.; Rothlisberger, U.; Grätzel, M. Origin of Unusual Bandgap Shift and Dual Emission in Organicinorganic Lead Halide Perovskites. Sci. Adv. 2016, 2, No. e1601156.

(13) Fang, H.-H.; Adjokatse, S.; Shao, S.; Even, J.; Loi, M. A. LongLived Hot-Carrier Light Emission and Large Blueshiftin Formamidinium Tin Triiodide Perovskites. Nat. Commun. 2018, 9, 243.

(14) Li, M.; Bhaumik, S.; Goh, T. W.; Kumar, M. S.; Yantara, N.; Grätzel, M.; Mhaisalkar, S.; Mathews, N.; Sum, T. C. Slow Cooling and Highly Efficient Extraction of Hot Carriers in Colloidal Perovskite Nanocrystals. Nat. Commun. 2017, 8, 14350.

(15) Grimaldi, G.; Crisp, W. R.; Ten Brinck, S.; Zapata, F.; Van Ouwendorp, M.; Renaud, N.; Kirkwood, N.; Evers, W. H.; Kinge, S.; Infante, I.; Siebbeles, D. A.L.; Houtepen, A. J. Hot-Electron Transfer in Quantum-Dot Heterojunction Films. Nat. Commun. 2018, 9, 2310.

(16) Guo, Z.; Wan, Y.; Yang, M.; Snaider, J.; Zhu, K.; Huang, L. Long-Range Hot-Carrier Transport in Hybrid Perovskites Visualized by Ultrafast Microscopy. Science 2017, 356, 59-62.

(17) Papagiorgis, P.; Protesescu, L.; Kovalenko, M. V.; Othonos, A.; Itskos, G. Long-Lived Hot Carriers in Formamidinium Lead Iodide Nanocrystals. J. Phys. Chem. C 2017, 121, 12434-12440.
(18) Kovalenko, M. V.; Protesescu, L.; Bodnarchuk, M. I. Properties and Potential Optoelectronic Applications of Lead Halide Perovskite Nanocrystals. Science 2017, 358, 745-750.

(19) Akkerman, Q. A.; Rainò, G.; Kovalenko, M. V.; Manna, L. Genesis, Challenges and Opportunities for Colloidal Lead Halide Perovskite Nanocrystals. Nat. Mater. 2018, 17, 394-405.

(20) Huang, H.; Polavarapu, L.; Sichert, J. A.; Susha, A. S.; Urban, A. S.; Rogach, L. A. Colloidal Lead Halide Perovskite Nanocrystals: Synthesis, Optical Properties and Applications. NPG Asia Mater. 2016, 8, No. e328.

(21) Fang, H.-H.; Raissa, R.; Abdu Aguye, M.; Adjokatse, S.; Blake, G. R.; Even, J.; Loi, M. A. Photophysics of Organic-Inorganic Hybrid Lead Iodide Perovskite Single Crystals. Adv. Funct. Mater. 2015, 25, 2378-2385.

(22) Saran, R.; Heuer Jungemann, A.; Kanaras, A. G.; Curry, R. J. Giant Bandgap Renormalization and Exciton-Phonon Scattering in Perovskite Nanocrystals. Adv. Opt. Mater. 2017, 5, 1700231.

(23) Butkus, J.; Vashishtha, P.; Chen, K.; Gallaher, K. J.; Prasad, K. K. S.; Metin, D. Z.; Laufersky, G.; Gaston, N.; Halpert, J. E.; Hodgkiss, J. M. The Evolution of Quantum Confinement in $\mathrm{CsPbBr}_{3}$ Perovskite Nanocrystals. Chem. Mater. 2017, 29, 3644-3652.

(24) Papagiorgis, P.; Manoli, A.; Protesescu, L.; Achilleos, C.; Violaris, M.; Nicolaides, K.; Trypiniotis, T.; Bodnarchuk, M. I.; Kovalenko, M. V.; Othonos, A.; Itskos, G. Efficient Optical Amplification in the Nanosecond Regime From Formamidinium Lead Iodide Nanocrystals. ACS Photonics 2018, 5, 907-917.

(25) Nah, S.; Spokoyny, B. M.; Soe, C. M. M.; Stoumpos, C. C.; Kanatzidis, M. G.; Harel, E. UltrafastImaging of Carrier Cooling in Metal Halide Perovskite Thin Films. Nano Lett. 2018, 18, 10441048.

(26) Fang, H.-H.; Protesescu, L.; Balazs, D. M.; Adjokatse, S.; Kovalenko, M. V.; Loi, M. A. Exciton Recombination in Formamidinium Lead Triiodide: Nanocrystals Versus Thin Films. Small 2017, 13, 1700673.

(27) Eperon, G. E.; Jedlicka, E.; Ginger, D. S. Biexciton Auger Recombination Differs in Hybrid and Inorganic Halide Perovskite Quantum Dots. J. Phys. Chem. Lett. 2018, 9, 104-109.

(28) Makarov, N. S.; Guo, S.; Isaienko, O.; Liu, W.; Robel, I.; Klimov, V. I. Spectral and Dynamical Properties of Single Excitons, Biexcitons and Trions in Cesium-Lead-Halide Perovskite Quantum Dots. Nano Lett. 2016, 16, 2349-2362.

(29) Katayama, T.; Suenaga, H.; Okuhata, T.; Masuo, S.; Tamai, N. Unraveling the Ultrafast Exciton Relaxation and Hidden Energy State in $\mathrm{CH}_{3} \mathrm{NH}_{3} \mathrm{PbBr}_{3}$ Nanoparticles. J. Phys. Chem. C 2018, 122, 52095214.

(30) Lyon, S. A. Spectroscopy of Hot Carriers in Semiconductors. J. Lumin. 1986, 35, 121-154.

(31) De Mello, J. C.; Wittmann, H. F.; Friend, R. H. An Improved Experimental Determination of External Photoluminescence Quantum Efficiency. Adv. Mater. 1997, 9, 230-232. 